\title{
Comparison of Snellen acuity, VER acuity, and Arden grating scores in macular and optic nerve diseases
}

\author{
HAROLD W. SKALKA \\ From the Combined Program in Ophthalmology, University of Alabama in Birmingham \\ Eye Foundation Hospital, Birmingham, Alabama, USA
}

SUMMARY Patients with various macular and optic nerve abnormalities underwent Snellen acuity, transient VER acuity, and Arden grating testing. Snellen acuity was the coarsest of the 3 evaluations, generally falling after Arden scores and VER acuity had already undergone significant degradation. The Arden gratings appeared to be the most sensitive of the 3 tests, equalling VER performance in optic nerve diseases and surpassing it in macular diseases. Variations in results between the different tests are generally understandable if one considers the functions tested by each and the anatomical derangements caused by the diseases in question. The Arden grating test appears to be an excellent and sensitive screening test for central visual disturbances.

Visual function has classically been defined by Snellen acuity, supplemented by visual field testing. Indeed, our definitions of legal blindness are couched in terms of Snellen acuity level or visual field subtended. These subjective tests have served, as they are easy to standardise and require a minimum of instrumentation. They may perhaps be taken as measures of the 'quantity' of vision. More difficult to standardise, and until recently much more difficult to evaluate, are estimations of the 'quality' of vision. Parameters such as contrast sensitivity at different spatial frequencies (a modulation transfer function) would provide one perspective on this subject. The recently developed Arden gratings ${ }^{1}$ provide a practical clinical method of evaluating such visual sensitivity.

A person's visual perception of the world may be altered in various ways by disease states. Optic nerve and macular diseases lead to visual disturbances that are often (in optic nerve disease) or usually (in macular disease) not relevent to description in visual field terminology. Likewise, macular and optic nerve disturbances may lead to significant functional loss which is not accurately reflected by tests of high-contrast resolution such as Snellen optotypes.

Snellen optotypes, VER responses to alternating checkerboard stimuli, and Arden contrast sensitivity grating charts are not equivalent visual stimuli.

Correspondence to Harold W. Skalka, MD, 1720 Eighth Avenue, South, Birmingham, Alabama 35233, USA.
Contrast, visual field subtended, and dynamism of presentation differ among these tests, which are measuring somewhat different visual functions. 'Acuity' according to each of these parameters, while hopefully showing congruence in health, would be surprising in disease. Indeed, we have found wide variations between Snellen acuity, VER acuity, and Arden scores in patients with optic nerve and macular diseases, variations which for the most part are predictable from consideration of the functions being evaluated and the pathology under evaluation.

\section{Materials and methods}

Patients with macular or optic nerve diseases were examined wearing their refractive corrections, including near additions where appropriate. Snellen acuity was recorded in the conventional manner, utilising a projected image at a distance of $20 \mathrm{ft}$ $(6 \mathrm{~m})$ in a darkened room. Arden grating scores were obtained under standardised lighting conditions ( $7 \mathrm{ft}$-candles), all tests being performed by the same examiner to ensure uniformity of test administration.

VER 'acuities' were obtained monocularly with monopolar leads placed $1 \mathrm{~cm}$ above the inion, referenced to the ipsilateral mastoid process, and recorded with the Nicolet CA-1000 system with television pattern generator. High-contrast black and white alternating check stimuli were presented at an alternation rate of $3.75 \mathrm{~Hz}$, and 128 transient 
responses covering the $200 \mathrm{~ms}$ following each alternation were averaged. At our testing distance the entire screen subtended $10^{\circ} 42^{\prime}$. horizontally and $8^{\circ} 1^{\prime} 30^{\prime \prime}$ vertically. While it is known that the latency of the recorded VER responses increases as the limits of resolution are approached, ${ }^{23}$ the major change which occurs is a reduction in amplitude of the recorded VER. ${ }^{4}$ We were interested in the limit of resolution rather than a 'normal versus abnormal' differentiation, and therefore end points were taken to be the smallest checks yielding recognisable responses (check sizes were reduced stepwise by half). Latencies did in fact increase as the patterns became more difficult for the patients to discern.

Some patients did not produce detectable VER responses to our patterned stimuli, and in these patients it was necessary to use light flash (luminance) stimuli in order to elicit a recordable VER. The 3 intensities of luminance used, in increasing order of brightness, were 'on-off' (the entire television screen flashing on and off), and the $S_{1}$ and $S_{16}$ settings on the Grass $\mathrm{PS}_{22}$ photostimulator with the stimulating light placed $4 \mathrm{ft}(1.2 \mathrm{~m})$ from the subject. These light flash (luminance) VERs, while obviously not measuring precisely the same function as the constant-luminance pattern reversal VERs, were recorded and averaged in an identical manner; 128 transient responses obtained from a monopolar electrode placed $1 \mathrm{~cm}$ above the inion were averaged for each monocular recording.

Our patient sample included a variety of macular and optic nerve diseases. Among these were senile macular degeneration, macular dystrophies, cystoid macular oedema, premacular fibrosis, retrobulbar neuritis, ischaemic optic neuropathy, traumatic neuropathies, tumours, etc. Patients with significant medial opacities, for example, cataracts, corneal scars, etc., were excluded. Patients ranged in age from 8 to 86 years, $75 \%$ being between 40 and 80 years old.

\section{Results}

In general, Snellen acuity proved most resistant to degradation by less severe macular and optic nerve disease states. This familiar test, using test symbols intimately known to patients and presented for recognition in high contrast at the patient's own chosen rate of response, did not prove to be a good screening test for macular or optic nerve diseases.

VER responses to alternating high-contrast black and white checks, obviating need for conscious recognition, interpretation, or ability to use clues (for example, 'could be E, B, O, or D, but certainly isn't $\mathbf{L}$ or $\mathbf{P}$ or $T$ ') were intermediate in sensitivity.
While Snellen acuity was sometimes more sensitive than VER acuity in macular diseases, $44 \%$ of examined patients showing relatively greater decrease in Snellen acuity (Fig. 1), the VER was definitely a more sensitive test in optic nerve diseases, where $82 \%$ showed greater abnormality on VER testing (Fig. 2). The superiority of the VER in comparison to Snellen testing in optic nerve disease has been shown by many investigators ${ }^{5-7}$ using delayed VER responses as the criterion of abnormality. The current study provides evidence that the same is true using loss of recordable VER responses to

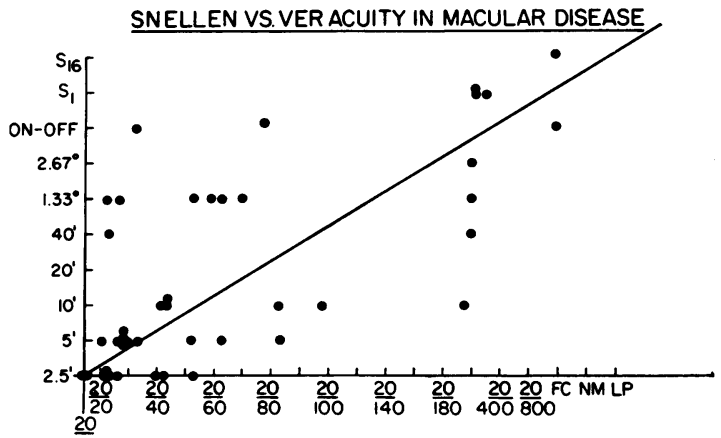

Fig. 1 Snellen acuity (abscissa) compared with VER acuity in patients with various macular diseases. On-off, $S_{1}$, and $S_{16}$ on ordinate scale (Figs. 1, 2, 5, and 6) refer to luminance VER stimuli (recorded for patients in whom no detectable response was obtained to large check pattern-reversal stimuli). In this and following figures the diagonal line runs from good acuity or score (lower left) to very poor acuity or score (upper right). $56 \%$ of data points lie above the diagonal, representing relatively greater degradation of VER acuity, while $44 \%$ (below the diagonal) show relatively greater fall-off in Snellen acuity.

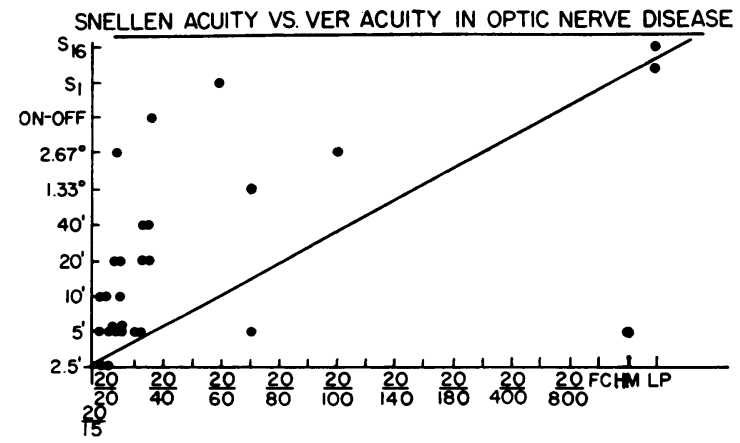

Fig. 2 Snellen acuity (abscissa) compared with VER acuity in patients with optic nerve diseases. $82 \%$ of data points lie above diagonal, showing relatively greater sensitivity of VER acuity to diseases of the optic nerves. 
SNELLEN ACUITY VS. ARDEN SCORE IN MACULAR DISEASE

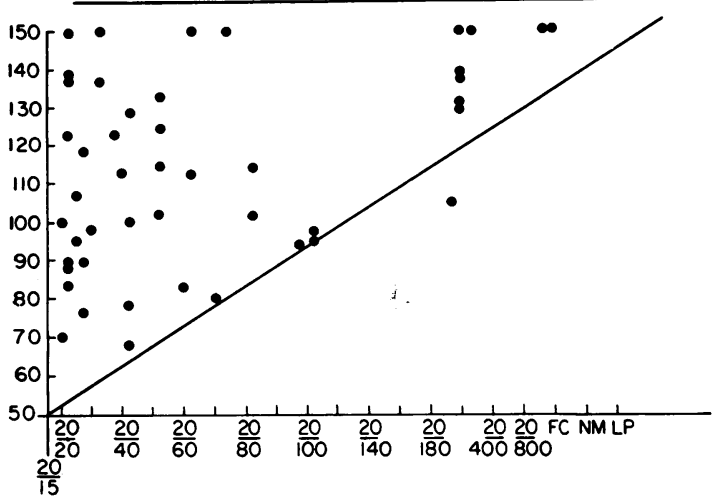

Fig. 3 Snellen acuity (abscissa) compared with Arden score in patients with macular diseases. Clear superiority of Arden test as indicator of macular disease can be seen, with $98 \%$ of data points above the diagonal line, representing relatively greater decline of contrast sensitivity in these patients. Note marked reduction of Arden sensitivity in many patients with retention of excellent Snellen acuity.

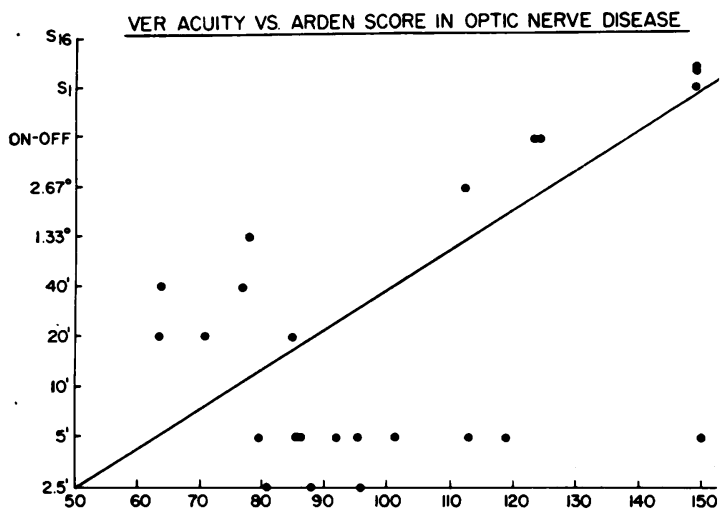

Fig. 5 Arden score (abscissa) compared to VER acuity in optic nerve disease patients. $50 \%$ of data points lie on each side of diagonal, demonstrating no superiority of one over the other test in optic nerve diseases.

pattern-reversal stimuli (or luminance stimuli when necessary) as the end-point criterion.

Arden contrast sensitivity gratings, an unfamiliar test of varying low contrasts requiring a rapid response (on penalty of a poorer score) proved on the whole to be the most discriminating of the 3 testing procedures in detecting abnormalities. While far better than Snellen acuity in both macular and optic nerve diseases, where virtually $100 \%$ of patients demonstrated relatively greater embarrassment of the Arden function (Figs. 3 and 4), the Arden test was equal to VER determinations in
SNELLEN ACUITY VS. ARDEN SCORE IN OPTIC NERVE DISEASE

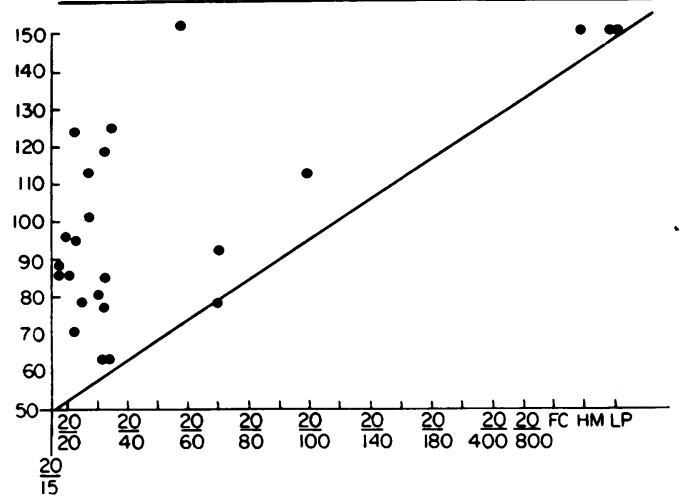

Fig. 4 Snellen acuity (abscissa) compared with Arden score in optic nerve disease patients. Superiority of Arden tests as indicator of optic nerve disease clearly visible, with no data points below diagonal. In all optic nerve disease eyes studied there was relatively greater impairment of the Arden score as compared to Snellen acuity.

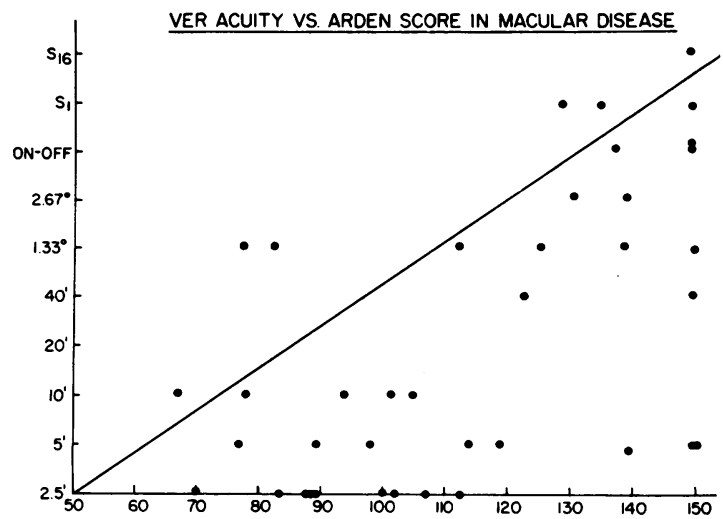

Fig. 6 Arden score (abscissa) compared to VER acuity in macular disease patients. $85 \%$ of data points lie below diagonal, demonstrating greater sensitivity of the Arden grating test in detecting macular disease.

optic nerve disease (Fig. 5) and was easily superior to VER acuity as an early indicator of macular dysfunction, where $85 \%$ of patients showed relatively greater impairment of Arden score than reduction of VER acuity (Fig. 6).

\section{Discussion}

VER responses, not surprisingly, seemed more sensitive to diseases of the optic nerves (transmission problems). For example, a 22-year-old female with a small central scotoma and Marcus-Gunn pupil 


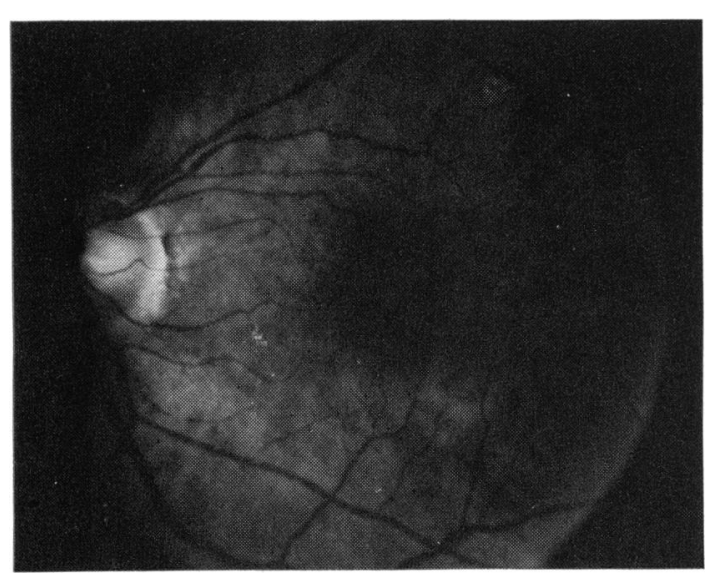

Fig. 7 Fundus photo OS of patient with surface wrinkling retinopathy.

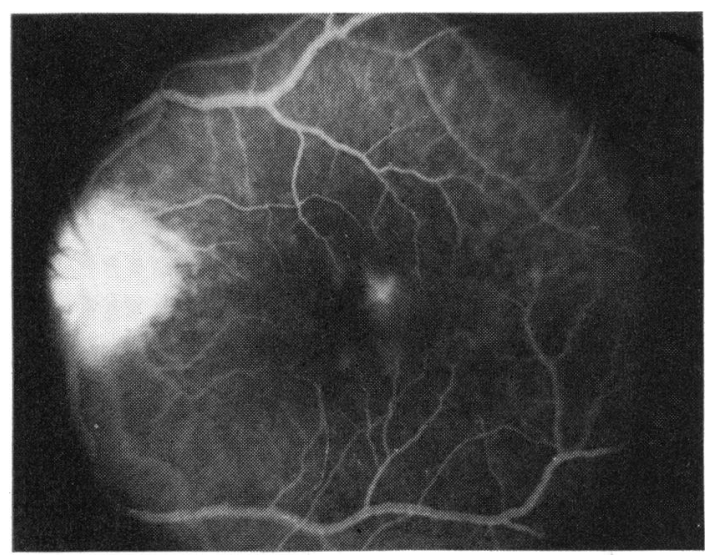

Fig. 8 Late-phase (10 minute) fluorescein angiogram of patient 6 weeks postcataract extraction with resolving cystoid macular oedema. Slight residual leakage can be seen.

OD secondary to a pituitary adenoma was noted to have $20 / 30$ - Snellen acuity OD, 20/20 OS. Arden grating scores were $63.5 \mathrm{OD}, 62.5 \mathrm{OS}$-both normal. VER responses showed poor voltage OU, with responses detectable to $20^{\prime}$ checks OS, but not OD. Another patient, a 39-year-old male with resolving retrobulbar neuritis OS, had Snellen acuities of 20/20 OD, 20/40+ OS; with Arden scores of $66 \mathrm{OD}, 125$ OS (being completely unable to distinguish the gratings on plates 5-7 OS). VER testing was normal with $2.5^{\prime}$ checks OD, but showed no detectable response OS to $80^{\prime}$ checks, and a delayed response to the stimulator screen light going on and off.

While Snellen acuity measurements seemed as useful as VER acuity measurements in macular diseases (Fig. 1), both paled in comparison with Arden test results in early detection of macular (image detection) dysfunction. For example, a 71-year-old female had surface wrinkling retinopathy OU (Fig. 7). Snellen acuity was 20/30 -2 OD, $20 / 60$ OS. VER responses could be obtained to $5^{\prime}$ checks with either eye. However, the lower contrast of the Arden gratings was completely nondiscernible with either eye (score of $150 \mathrm{OU}$ ).

'Denser' anatomical lesions such as the macular degenerations tended to affect VER acuity as well as Arden scores. For example, a 54-year-old male was 6 weeks after cataract extraction OS, with resolving cystoid macular oedema demonstrated by fluorescein angiography (Fig. 8). Snellen acuity had improved to $20 / 25+3$. VER responses were small with $40^{\prime}$ checks, and were nonrecordable with $20^{\prime}$ checks. The Arden score was 123 (plates 6 and 7 were completely missed).

Focal foveal photoreceptor abnormalities would be expected to cause more interference with VER and Snellen acuities (both tests of resolution of macular targets) than with sinusoidally distributed

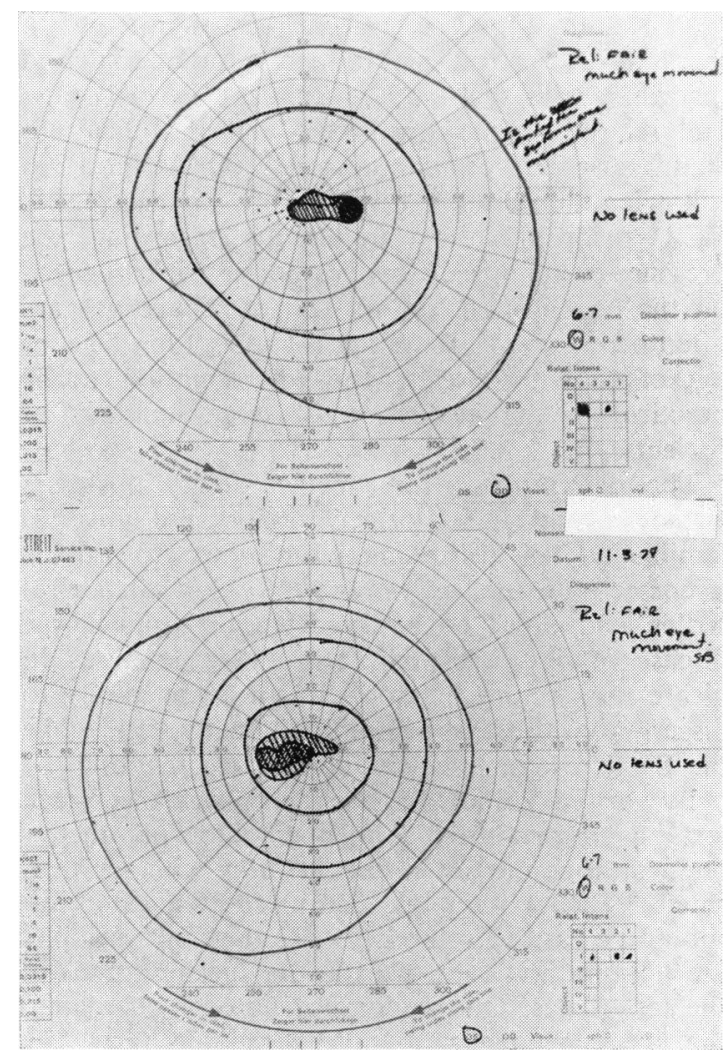

Fig. 9 Visual fields of patient with central form of cone dystrophy, showing discrete central scotomas. 
grating contrast changes over the posterior pole, and again, clinical experience tended to confirm this. For example, an 8-year-old with the central form of cone dystrophy made 483 errors on the Farnsworth 100-hue test and had variably recorded Snellen acuities of 20/80-20/200 OD and 20/7020/100 OS. On VER testing poor responses were obtained to $80^{\prime}$ checks with either eye. The central scotomas present (Fig. 9) raised his Arden scores only slightly, to $82.5 \mathrm{OD}, 77.5 \mathrm{OS}$.

Disease cephalad to the occipital cortex should have less effect on VER results recorded at the occipital pole than on subjective responses requiring conscious interpretation and reporting (as would also be true in cases of hysteria or malingering). For example, a 13-year-old female with neurological sequelae of bacterial meningitis in childhood had slight nystagmus, right esotropia, and temporal disc pallor with a mildly amaurotic pupil OD. Snellen acuity was hand movements at $4 \mathrm{ft}(1.2 \mathrm{~m}) \mathrm{OD}$, 20/70 OS. Arden gratings were not detectable OD (score 150) and scored at 92 OS. However, good VER responses to $5^{\prime}$ checks could be recorded using either eye.

VER stimuli may be varied almost infinitely with the aid of electronics. However, stimulating and recording with such apparatus is costly, elaborate, and relatively time-consuming, and is difficult to standardise between laboratories. Furthermore, in following diminishing responses to an end point, VER recordings are ambiguous regarding the presence or absence of small responses superimposed on the background 'noise', and extrapolations are often necessary. VER testing is simply too demanding of time, resources, and interpretation to be practical in the evaluation of large numbers of patients.

The determination of Snellen acuity possesses virtues beyond its ease of performance and universal familiarity. This test requires the subject to provide a correct answer, as opposed to the categorical Arden end point ('yes, I see it'). In following a disease process over time the patient anxious to please his doctor and do well can claim ability to discern the gratings before he actually can, while the Snellen letters require correct elucidation.

By varying stimulus size and contrast the Arden test has incorporated 2 of the major parameters one would be interested in evaluating with sophisticated VER stimulators. The Arden grating test should be easy to standardise, as the only variables are lighting and rate of exposure (presentation) of the test plates. One factor not noted by Arden is the age dependence of 'normal' scores $^{8}$ (Fig. 10), and this must be taken into account (as was done in the present study) when evaluating a subject's

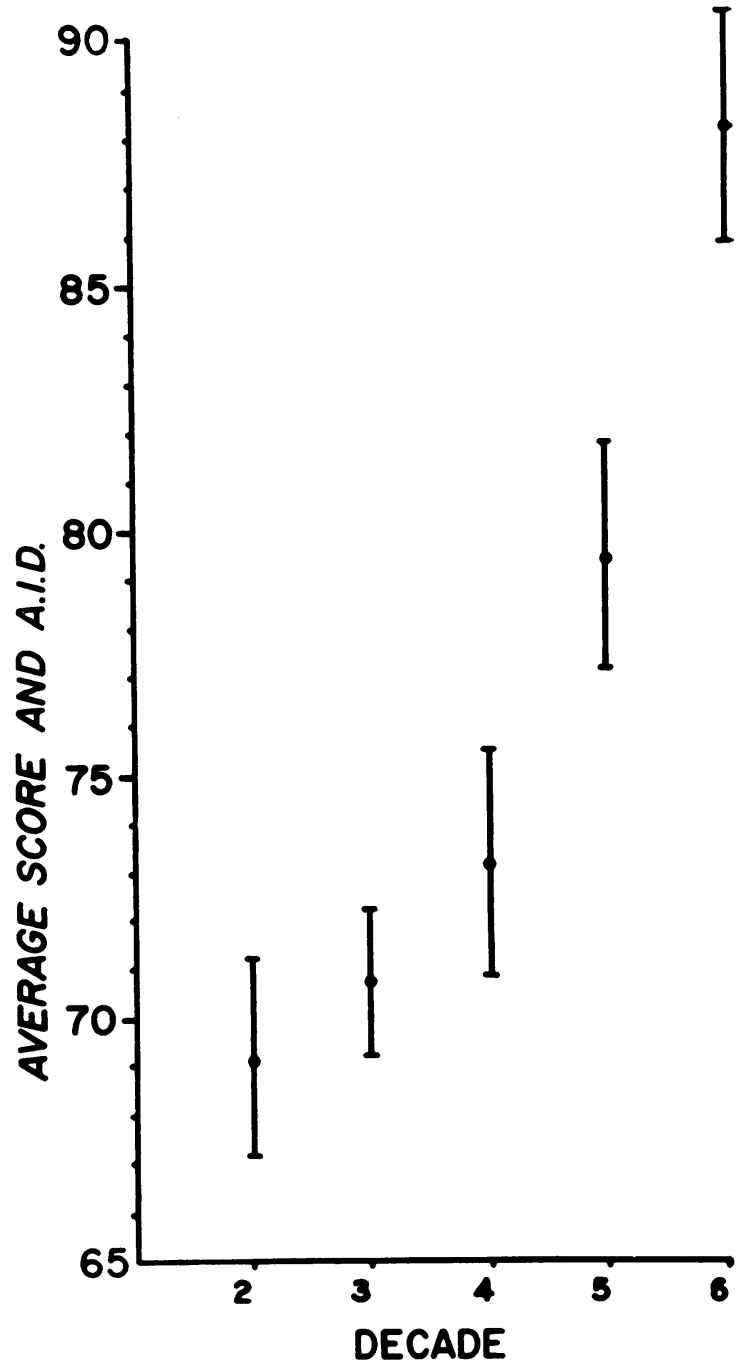

Fig. 10 Average Arden grating scores and average interocular differences (AID) of 100 normal patients grouped by age in decades.

test performance. A simply performed and interpreted clinical test, the Arden gratings appear to offer a sensitive screening test for a host of subtle abnormalities.

\section{References}

${ }^{1}$ Arden GB. The importance of measuring contrast sensitivity in cases of visual disturbances. $\mathrm{Br} J$ Ophthalmol 1978;62: 198-208.

'Perry NW Jr, Childers DG. The Human Visual Evoked Response. Springfield, Illinois: Charles Thomas, 1969;35.

${ }^{3}$ Sokol S, Jones K. Implicit time of pattern evoked potentials in infants: an index of maturation of spatial vision. Vision Res 1979;19:747-755. 
4Millodot M, Riggs LA. Refraction determined electrophysiologically: responses to alteration of visual contours. Arch Ophthalmol 1970;84:272-278.

${ }^{5}$ Halliday AM, McDonald WI, Mushin J. Visual evoked response in diagnosis of multiple sclerosis. $\mathrm{Br}$ Med $J$ 1973; 4:661-664.

- Milner BA, Regan D, Heron JR. Differential diagnosis of multiple sclerosis by visual evoked potential recording. Brain 1974;97:755-772.

'Arden GB, Gucukoglu AG. Grating test of contrast sensitivity in patients with retrobulbar neuritis. Arch Ophthalmol 1978;96:1626-1629.

8Skalka HW. The effect of age on Arden grating acuity. Br J Ophthalmol (in press). 